(C) 1999 IEEE. Personal use of this material is permitted. Permission from IEEE must be obtained for all other uses, in any current or future media, including reprinting/republishing this material for advertising or promotional purposes, creating new collective works, for resale or redistribution to servers or lists, or reuse of any copyrighted component of this work in other works. 


\title{
Depth Identification Accuracy of a Three Layer Phoswich PET Detector Module
}

\author{
J. Seidel, J. J. Vaquero, Member, IEEE, S. Siegel ${ }^{1}$, Member, IEEE, \\ W. R. Gandler", Senior Member, IEEE, and M. V. Green \\ Nuclear Medicine Department, Clinical Center \\ "Center for Information Technology \\ National Institutes of Health, Bethesda, Maryland 20892
}

\begin{abstract}
We describe a PET detector module that provides three levels of depth-of-interaction (DOI) information. The detector is a $9 \times 9$ array of $2 \mathrm{~mm} \times 2 \mathrm{~mm} \times 12 \mathrm{~mm}$ deep phoswich crystal elements, each consisting of $4 \mathrm{~mm}$ long LSO (entrance layer), GSO (middle layer) and BGO (exit layer) crystals joined optically together end-to-end. The BGO exit layer is directly coupled to a miniature position-sensitive photomultiplier tube (PSPMT). Delayed charge integration, a method that exploits differences in the light decay times of these scintillators, is used to determine the layer-of-interaction. DOI accuracy, measured by scanning a slit source of $511 \mathrm{keV}$ radiation along the length of the module was $86 \%$ for the LSO layer, $80 \%$ for the GSO layer and $84 \%$ for the BGO layer. Energy resolution at $511 \mathrm{keV}$ was $19 \%$ for LSO, $21 \%$ for GSO and $40 \%$ for BGO. Apparent gain differed between layers in the ratios 2.7:1.9:1.0 (LSO:GSO:BGO). Crystal separation was good between crystals in the LSO layer, acceptable between crystals in the GSO layer and poor between crystals in the BGO layer due, primarily, to the pronounced spatial non-linearity of the PSPMT. The delayed charge integration method, however, does appear suitable for obtaining multi-level depth information when DOI effects are particularly significant, e.g. in very small ring diameter PET scanners for small animal imaging.
\end{abstract}

\section{INTRODUCTION}

PET detector modules with depth-of-interaction (DOI) capability improve spatial resolution and resolution uniformity by more accurately locating the endpoints of lines-of-response in space [1]-[11]. Moreover, a DOI detector module can also increase the number of axial slices in a "side-looking" PET scanner, a ring of detectors in which annihilation radiation enters through the side of a crystal array, rather than the front. Here, we evaluated a phoswich detector module in which three levels of DOI information are obtained by exploiting differences in light decay time between three different scintillators, LSO, GSO and BGO.

\section{MATERIALS AND METHODS}

\section{A. Detector Module}

The detector module was comprised of three layers of individual crystals (Figure 1). Each layer was geometrically identical: $9 \times 9$ arrays of $2 \mathrm{~mm} \times 2 \mathrm{~mm}$ crystals $(2.2 \mathrm{~mm}$

\footnotetext{
' Current address: Concorde Microsystems, 10427 Cogdill Rd, Suite 500, Knoxville, Tennessee, 37932
}

pitch), each $4 \mathrm{~mm}$ long. The entrance layer was comprised of $\mathrm{Lu}_{2} \mathrm{SiO}_{5}: \mathrm{Ce}$ (LSO) crystals (CTI, Knoxville, TN), the middle layer of $\mathrm{Gd}_{2} \mathrm{SiO}_{5}: \mathrm{Ce}$ (GSO) crystals (Hitachi Chemical Co., Ltd., Ibaraki, Japan) and the exit layer of $\mathrm{Bi}_{4} \mathrm{Ge}_{3} \mathrm{O}_{12}$ (BGO) crystals (Rexon, Beachwood, $\mathrm{OH}$ ). The exit array was coupled with optical grease (type V-780, Visilox, Troy, NY) to the face of a Hamamatsu R5900-C8 position-sensitive photomultiplier tube (PSPMT). The LSO and BGO crystal elements were mechanically ground and polished on all sides with the exception of the entrance ends of the LSO crystals which were diffusely ground (3D Precision Optics, Ravenna, $\mathrm{OH}$ ). The GSO crystals were chemically etched on all surfaces. Each LSO/GSO/BGO phoswich element was assembled individually by permanently joining an LSO and BGO crystal to a GSO crystal with transparent cyanoacrylate glue. Each assembled phoswich element was then double-wrapped with diffusely reflective Teflon tape. Eighty-one of these finished elements were packed together to form a $9 \times 9$ array.

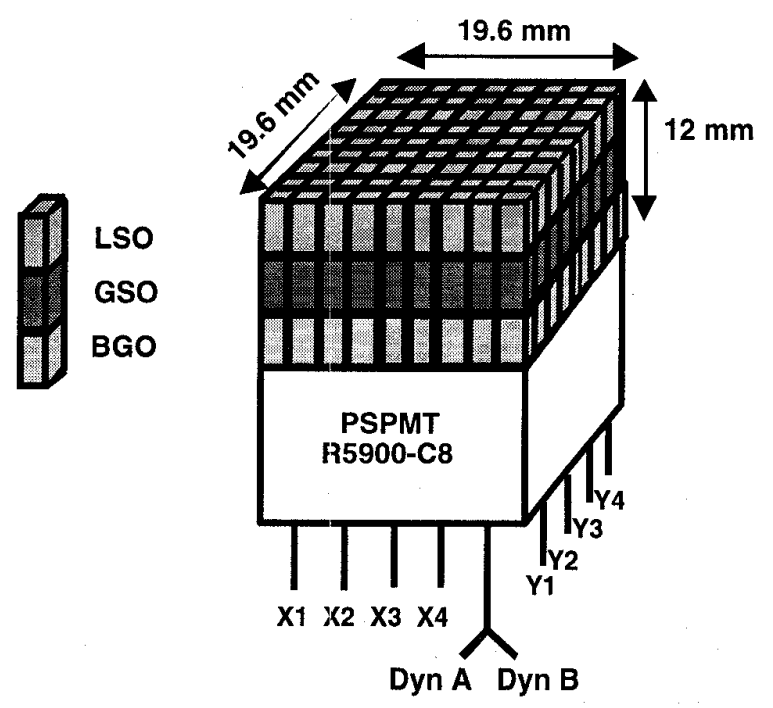

Figure 1. Phoswich detector module assembly.

\section{B. Electronics, Data Acquisition and Processing}

The R5900-C8 PSPMT contains four X anode plates and four $\mathrm{Y}$ anode plates that collect the amplified charge generated by each scintillation event. These 8 anode signals were fed to FERA 4300B charge-integrating analog-to-digital converters (LeCroy Research Systerns, Chestnut Ridge, NY), digitized and these digital values used in a standard centroid algorithm to calculate the $X, Y$ position of the event within the field-ofview of the tube. 
The DOI, or layer-of-interaction, of the event is obtained by splitting the signal from the last dynode (Figure 1) into two parts. The first of these signals is manipulated such that this signal is fully integrated (Figure 2) by the FERA ADC, i.e. an integration window of width "W" is opened that sums the entire signal. The integration window for the second signal, on the other hand, is delayed with respect to the first signal such that when the window $\mathrm{W}$ opens, only the tail of the second signal is integrated. Depending on the delay " $\mathrm{D}$ " and width $\mathrm{W}$, the ratio of the delayed integral to the full integral (or the reverse) can be shown to depend on the light decay time of the scintillator. Since the light decay times of the three phoswich scintillators differ $(\mathrm{LSO}=40 \mathrm{~ns}, \mathrm{GSO}=60 \mathrm{~ns}, \mathrm{BGO}=300$ $\mathrm{ns}$ ), a plot of delayed/full (or full/delayed) ratios should distinguish between the three scintillators and, correspondingly, identify the layer-of-interaction.

\section{Full Charge Integration Delayed Charge Integration}
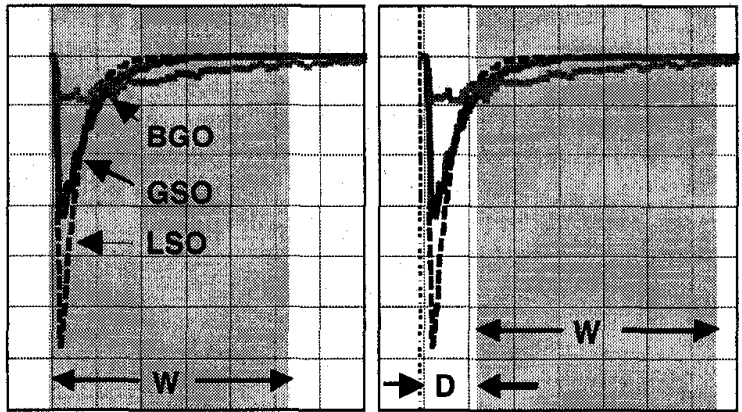

Figure 2. Illustration of the delayed charge integration method. Simulated pulses for LSO, GSO, and BGO are shown on a scale of $100 \mathrm{~ns}$ per division. The ADC gate is opened for charge integration for a period $\mathrm{W}$ as indicated. In the full charge integration case (left panel), the gate is triggered just in time before the pulse arrives. In the delayed charge integration case (right panel) the ADC gate is opened after a delay $\mathrm{D}$ with respect to the full integration case.

The minimum value of $\mathrm{W}$ was determined by plotting the magnitude of the full charge integral (or location of the photopeak maximum) against the width of the integration window for the three different scintillators (Figure 3, left panel). Starting with $W=400 \mathrm{~ns}$, the full charge integral of LSO does not change as $\mathrm{W}$ increases. This result is expected since LSO has a $40 \mathrm{~ns}$ light decay time and this signal will be nearly fully integrated by $200 \mathrm{~ns}$. Further increases in the window width should have no effect and none is observed. The full charge integral of GSO, on the other hand, increases slightly from $400 \mathrm{~ns}$ but becomes almost constant for $\mathrm{W}>600$ ns. This result is consistent with the presence of a faint $(10 \%$ of total), longer-lived ( $600 \mathrm{~ns}$ ) component in the light decay of GSO [12]. Finally, integration of the BGO signal would not be expected to be complete until well after $400 \mathrm{~ns}$ and the BGO integral continues to increase with increasing $W$ up to 1100 ns. However, since approximately $85 \%$ of the BGO signal has been integrated by $600 \mathrm{~ns}$ and both LSO and GSO are almost fully integrated, we chose $\mathrm{W}=600 \mathrm{~ns}$ as a reasonable minimum full charge integration window.

If the ratio of the delayed charge integral to full charge integral is plotted as a function of the delay, $D$, the graph in the right panel of Figure 3 is obtained. The observed ratios fall into 3 distinct bands, one for each scintillator. Inspection of this figure indicates that the separation between bands is not a strong function of $\mathrm{D}$ and the three scintillators can be distinguished from one another for all values of $D$ investigated (70 $190 \mathrm{~ns})$. We chose $\mathrm{D}=130 \mathrm{~ns}$.
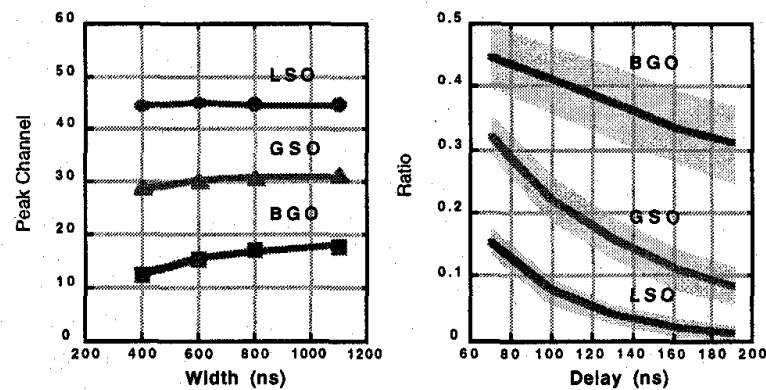

Figure 3. Variation of the photopeak location with the ADC gate width (left panel). The ratio of the delayed to the full charge integral as a function of delay is shown in the right panel. Gray areas in the right panel indicate one standard deviation variations.

If the detector module is illuminated by a distant ${ }^{18} \mathrm{~F}$ source, and the full charge integral plotted against the delayed charge integral for each event (using $D=130 \mathrm{~ns}$ and $W=600$ ns), one obtains the "phoswich diagram" shown in the left panel of Figure 4. This graph is an alternative means of expressing the same result shown in the right panel of Figure 3. Rather than the quasi-parallel bands in the figure, events occurring in each scintillator now appear as "spokes" radiating away from the origin. The spoke with the steepest slope corresponds to the "fastest" scintillator (LSO), while the spoke with the smallest slope corresponds to the "slowest" scintillator (BGO).
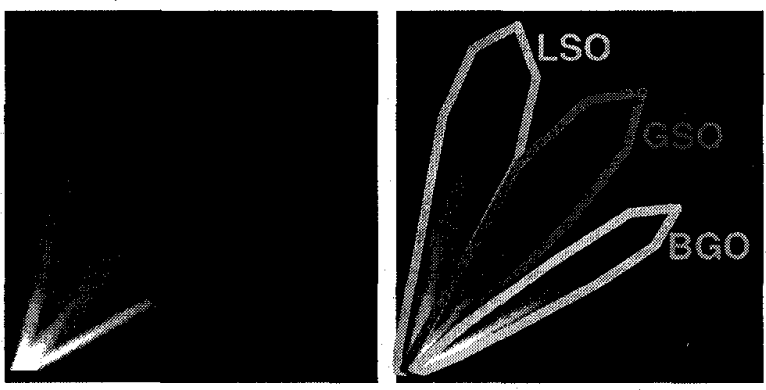

Figure 4. Phoswich diagrams: full charge integral (vertical axis) vs. delayed charge integral (horizontal axis). "Spokes" in left panel are the result of applying the delayed charge integration conditions to field flood data. Each spoke corresponds to a different scintillator. The right panel shows ROls defining LUT regions used for layer assignment.

The method by which an event is assigned to a scintillator layer is indicated in the right panel of Figure 4. Here, regionsof-interest (ROIs) have been (generously) drawn around each spoke. When an event occurs, the magnitudes of the delayed and full charge integrals for that event define a point in the phoswich diagram. If that point falls within the LSO regionof-interest, the event is assigned to the LSO layer, if within the 
GSO ROI, to the GSO layer, and if within the BGO ROI, to the BGO layer. Thus, the phoswich diagram defines a lookup table (LUT) for depth or layer assignment. This scheme and the ROIs shown in this figure were used in the experiments described below.

\section{DOI Accuracy}

In order to obtain an estimate of DOI accuracy, a highly collimated $1.5 \mathrm{~mm}$-wide slit source of ${ }^{18} \mathrm{~F}$ was stepped in 1 $\mathrm{mm}$ increments along the side of the detector module as shown in Figure 5. At each point, the number of events occurring in the layer being illuminated was determined and expressed as a fraction of events occurring anywhere in the module. For this experiment, the module was placed in time coincidence with a second detector in order to suppress the background count rate of ${ }^{176} \mathrm{Lu}\left(300 \mathrm{~s}^{-1} \mathrm{~cm}^{-3}\right.$ of LSO), a rate comparable to that generated by the collimated $511 \mathrm{keV}$ photon beam $\left(\approx 1,000 \mathrm{~s}^{-1}\right)$. This coincidence technique increased the signal-to-noise ratio of this measurement by a large factor.

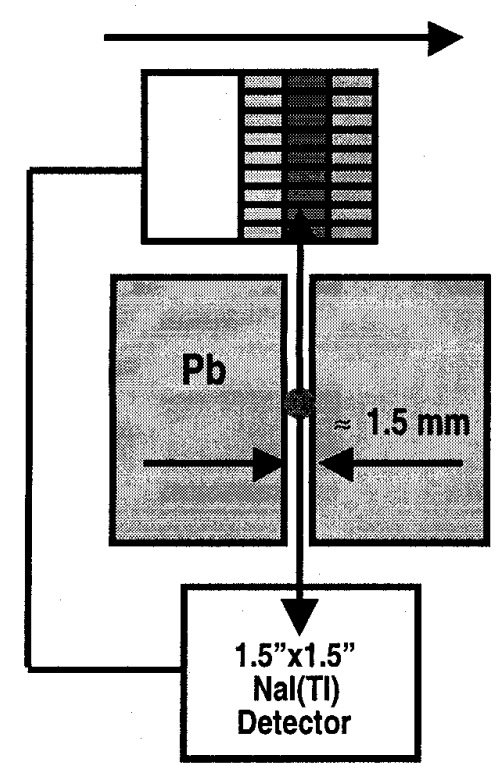

Figure 5. Geometry of the scanning slit source experiment. The phoswich detector is operated in time coincidence with a $\mathrm{NaI}(\mathrm{Tl})$ detector in order to suppress the intrinsic background caused by ${ }^{177} \mathrm{Lu}$ decays in the LSO layer.

Images of the field-of-view of each layer were also created from data acquired when the slit beam was centered on the side of a layer. These images allow a qualitative assessment of the degree to which the phoswich layer identification scheme places an event in the proper layer.

\section{Crystal Identification within a Layer}

Although the primary purpose of this work was to assess the DOI accuracy of the module, the ability of the module to resolve crystals within a layer is also important. Accordingly, we illuminated the module with a distant ${ }^{10} \mathrm{~F}$ source, applied the DOI algorithm to these data and created a field-flood image for each layer. The apparent separation between crystals in these images provides a qualitative measure of crystal identification accuracy in each layer.

\section{E. Spectral Resolution}

The radial direction in the spokes shown in the phoswich diagram is proportional to absorbed photon energy and each spoke should contain a bright region near its end representing photopeak events. However, in the phoswich diagram for the entire detector (Figure 4, left panel), no such bright photopeak region is seen in any of the spokes. This effect could be explained if spectra from each phoswich element have different gains. The superposition of such spectra would result in a "blurred" energy spectrum in which the photopeak region might not be identifiable. Accordingly, we examined the energy spectra created within individual phoswich elements at several different locations within the array in order to determine the degree to which apparent gain varied from one element to the next.

\section{RESULTS}

Images of each layer when the slit source is centered on the side of that layer are shown in Figure 6. The slit source is illuminating the module from the right side of the Figure. DOI accuracy ranges from $80 \%$ in the GSO layer to $86 \%$ in the LSO layer. Note the presence of an LSO crystal in the BGO layer (bottom right) and the absence of a LSO crystal in the LSO layer at the same location (upper right). A BGO crystal appears in the BGO image at this location when the LSO layer is illuminated (upper left), but may not be visible in the photographic reproduction of this figure. This LSO/GSO/BGO phoswich element was accidentally reversed during assembly of the module, interchanging the position of the two LSO and BGO crystals.

DOI accuracy is portrayed in Figure 7. Accuracy is defined as the fraction of events occurring in the illuminated layer compared to events occurring anywhere in the module.

The effect of applying the DOI algorithm to $511 \mathrm{keV}$ field flood data is shown in Figure 8. Without DOI information, illumination of the module yields a "sum" image, a composite of all events occurring anywhere in the module. When the DOI algorithm is applied, this image separates into three images, one for each layer. Crystals are best separated in the LSO layer, less well in the GSO layer and poorly in the BGO layer. Nonetheless, all crystals in all layers are evident, except for two small low gain regions in the upper and lower portions of the BGO image. It is likely that the low energy threshold set during the acquisition of these data was somewhat too high and some events occurring in these particularly low-gain regions were lost.

Phoswich diagrams for two individual phoswich elements (elements number 52 and 9 in the array) are shown in Figure 9. Photopeaks are clearly evident in each spoke of the diagram and are detectable even for an element in the corner of the array. Note that the radial magnitude of the spokes for element 52 is much greater (higher apparent gain) than for the corner element. 


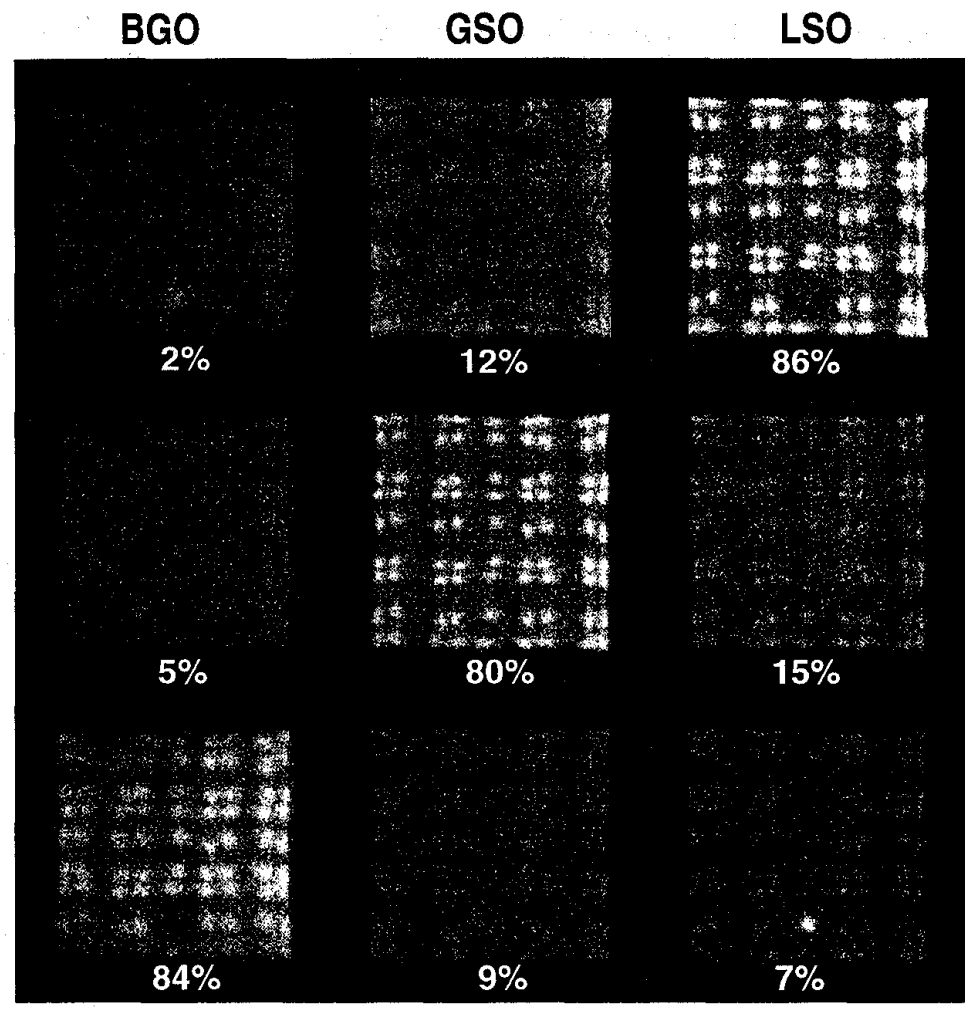

\section{Illuminated}

Layer:

LSO

GSO

BGO

Figure 6. Images resulting from side-illumination of each layer.

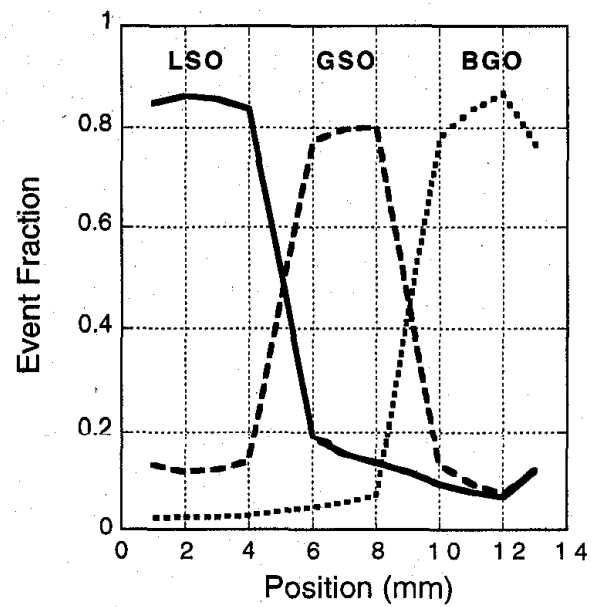

Figure 7. Fraction of events assigned to each layer when scanning a collimated slit source along the side of the detector module.
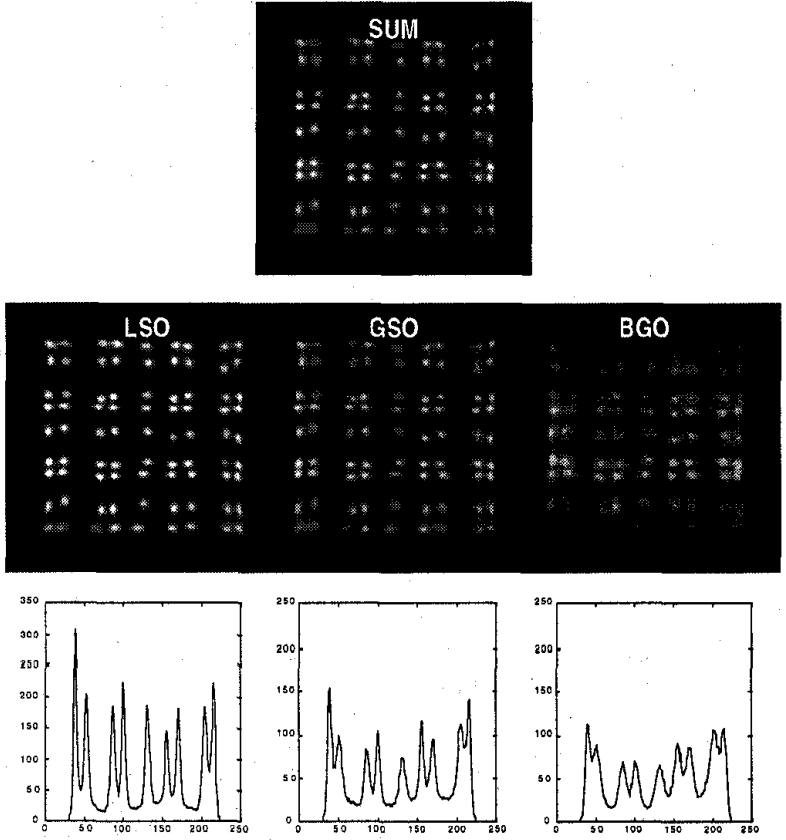

Fig.8 DOI decomposition of the sum field flood image into three layer images, and count profiles along the central row (bottom). 
If all events occurring in element 52 are sorted into an energy spectrum without regard to DOI information, the composite spectrum of these events appears as in the left panel of Figure 10. If the DOI conditions are applied, this spectrum separates into three component spectra, one for each scintillator (right panel). The photopeak maxima appear in the ratios: 2.7:1.9:1.0 (LSO:GSO:BGO) in this figure with corresponding energy resolutions of: $19 \%, 21 \%$ and $40 \%$. For element 9 , the photopeak maxima appear in the ratios: 2.0:1.7:1.0 with energy resolutions of $26 \%, 32 \%$ and $61 \%$, respectively.

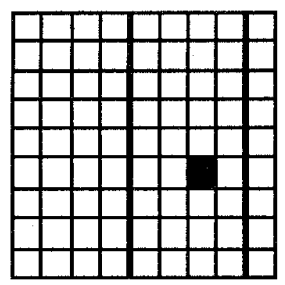

\#52

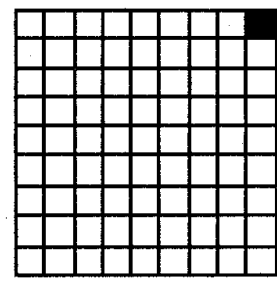

\#9

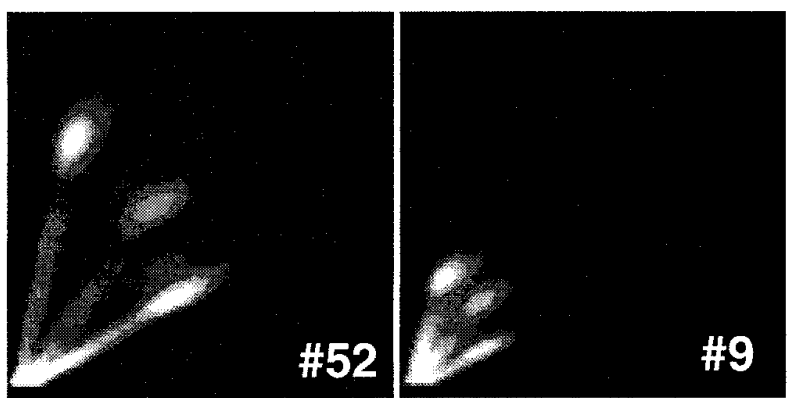

Figure 9. Phoswich diagrams for two phoswich elements in the array, element \#52 (left panel) and element \#9 (right panel). The locations of these elements within the array are indicated at the top of the figure.

\section{DISCUSSION}

The results obtained in the scanning slit source experiment (Figure 7) suggest that the delayed charge integration method identifies the layer-of-interaction with reasonable accuracy. More than $80 \%$ of events are correctly assigned to a layer when the (relatively wide) source beam falls onto the center of that layer. Moreover, this result was obtained without energy windowing and with LUTs defined on a global phoswich diagram (Figure 4 ) created by flood field illumination of the module.

An asymmetry can be seen in the DOI accuracy results portrayed in Figure 6 . When the beam illuminates the middle of the GSO layer, we would expect the same number of photons to scatter into the LSO layer as into the BGO layer. Since the absorption characteristics of LSO and BGO are similar, we would also expect to detect about the same number of events in each layer. Instead, we find more mis-positioned events in the LSO layer (15\%) than in the BGO layer $(5 \%)$. This difference may result from the use of a single global discriminator threshold rather than true energy windowing for each individual phoswich element. Since the light yield differs among the scintillators, a single, global threshold translates into three different effective energy thresholds for a given phoswich element. In particular, the effective energy threshold is highest for the BGO layer, and lowest for the LSO layer. Some scatter events may, therefore, fall below the threshold in the BGO layer whereas the same events would be detected if they took place in the LSO layer since the scintillation efficiency of LSO is higher.

Examination of Figure 9 suggests a strategy to implement energy windowing in a way that is likely to improve DOI accuracy significantly. Instead of defining the layer identification LUTs on a global phoswich diagram, the event selection criteria could be applied to 81 phoswich diagrams created for the individual phoswich elements. As seen in Figure 9, individual crystal photopeaks are clearly visible in each spoke, even at the corner of the array, so that LUTs defined on this diagram could immediately include energy discrimination for each individual scintillator in that element. Regions bounding each spoke could be drawn to include the photopeak only, or the photopeak and Compton edge of each spoke, thereby rejecting low energy or scatter events. If, in these same individual diagrams, the angular widths of the spokes were restricted by the LUTs, events occurring in the gaps between spokes could also be rejected. These events are comprised in part of intra-module scatter events in which energy is deposited in two different scintillator types. LUTs narrowly drawn around each spoke would tend to remove these events thereby improving DOI accuracy. Spatial windowing in each scintillator layer could also be applied to further reject intra-module scatter [13]. The combination of these rejection criteria that yields the greatest DOI and position detection accuracy while retaining the highest possible sensitivity remains to be determined.

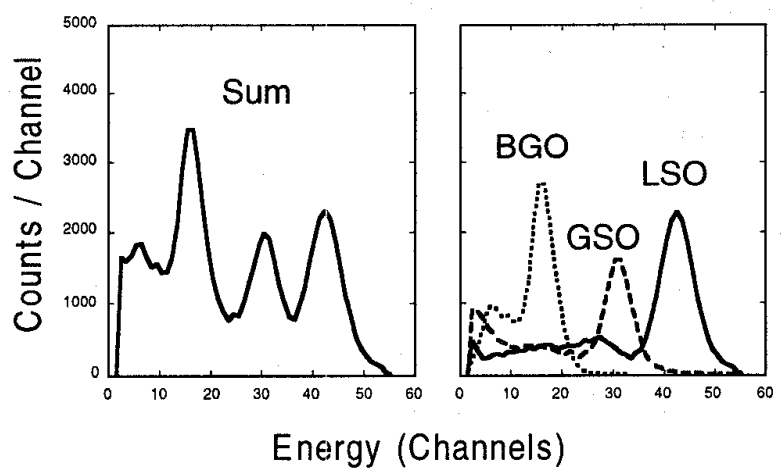

Figure 10. Sum energy spectrum (left panel) and DOI decomposition of this spectrum (right panel) for phoswich element \#52.

The inability of the global phoswich diagram to portray photopeaks in each spoke arises from the spatial gain variation of the R5900-C8 phototube. The apparent gain shown in phoswich element number 52 (Figure 9) is much larger (about three times larger) than the apparent gain in the corner phoswich element. Superposition of such apparent gain-varying spectra, one for each phoswich element, would be expected to produce a composite spectrum in which the individual photopeaks are blurred together and no single photopeak is detectable. This result is in accord with the observation that, while photopeaks are readily detectable in the spokes of individual phoswich elements, the spokes in the composite phoswich 
diagram (Figure 4) appear continuous in the radial direction and little, if any, structure is visible.

The energy spectra associated with individual phoswich elements provide some information as to the effectiveness of the transmission and collection of scintillation light within the module. In a three layer phoswich, it might be expected that significant light loss would occur since some or all of the scintillation light created within a crystal must, in general, pass across several interfaces, and through several crystals, with different optical properties in order to reach the photocathode. At each interface and in each medium, some of this light is lost so that the total loss could be substantial. However, inspection of Figure 10 indicates that photopeaks are clearly evident in the energy spectrum of a single phoswich element implying that a reasonable amount of light from each phoswich crystal does, in fact, reach the photocathode of the PSMPT. The energy resolution values for LSO and BGO determined from these spectra are approximately $30-40 \%$ poorer than would be observed if these same crystals were coupled individually and directly to the same kind of phototube [13]. The order in which the LSO, GSO and BGO crystals are combined may also affect energy resolution [5].

While light loss within the phoswich module might be acceptable in and of itself, this loss combines with the pronounced spatial non-linearity of the R5900-C8 PSPMT to impair identification of individual crystals in the BGO and GSO layers (Figure 8). For the "bright" scintillator LSO, the very narrow profile of each LSO crystal can be resolved even in the presence of the spatial non-linearity. In contrast, the increased widths of the profiles of BGO and GSO, and their forced clustering due to the non-linearity, obscures the separation between crystals in these layers. If this non-linearity were removed, and the spacing between peaks in the profiles shown in Figure 8 made regular, crystal identification would be improved, particularly for the GSO and BGO layers. Spatial linearity has, in fact, been improved in the newly developed Hamamatsu R5900-C12 [14] and may reduce or eliminate this problem.

\section{CONCLUSIONS}

Scintillation events occurring in a three layer phoswich detector module can be assigned to the correct layer of interaction with good accuracy using delayed charge integration, a method sensitive to differences in light decay time of the scintillators and independent of absolute pulse magnitude. A variety of event selection criteria are available that could potentially improve DOI and position detection accuracy, though with some reduction in sensitivity.

\section{ACKNOWLEDGEMENTS}

The authors thank Burton E. Chidakel for his expert assistance in preparing the mechanical and electrical components of the detector module. J. J. V. was supported, in part, by a grant from CICYT (Spanish Government). S. S. was supported by a grant from the National Research Council.

\section{REFERENCES}

[1] J.S. Karp, M.E. Daube-Witherspoon, "Depth-of-Interaction Determination in $\mathrm{Nal}(\mathrm{Tl})$ and $\mathrm{BGO}$ Scintillation Crystals Using a Temperature Gradient," Nucl Instr Meth, Vol. 260, pp. 509-517, October 1987

[2] P. Bartzakos, C.L. Thompson, "A Depth-encoded PET Detector," IEEE Trans Nucl Sci, Vol. 38, pp. 732-738, April 1991

[3] W.W. Moses, S.E. Derenzo, C.L. Melcher, R.A. Manente, "A Room Temperature LSO/PIN Photodiode PET Detector Module That Measures Depth of Interaction," IEEE Trans Nucl Sci, Vol. 42, pp. 1085-1089, August 1995

[4] C. Moisan, G. Tsang, J.G. Rogers, E.M. Hoskinson, "Performance Studies of a Depth Encoding Multicrystal Detector for PET," IEEE Trans Nucl Sci, Vol. 43, pp. 1926-1931, June 1996

[5] A. Saoudi, D. Rouleau, R. Lecomte, M. Andreaco, M. Casey, R. Nutt, H. Dautet, "Performance Studies of Depth Encoding Multicrystal Detectors Using Avalanche Photodiodes," J Nucl Med, Vol. 39, p. 92P, May 1998

[6] L.R. MacDonald, M. Dahlbom, "Depth of Interaction for PET Using Segmented Crystals," IEEE Trans Nucl Sci, Vol. 45, pp. 2144-2148, August 1998

[7] R.S. Miyaoka, T.K. Lewellen, H. Yu, and D.L. MacDaniel, "Design of a Depth of Interaction (DOI) PET Detector Module," IEEE Trans Nucl Sci, Vol. 45, pp. 1069-1073, June 1998

[8] S. Yamamoto and H. Ishibashi, "A GSO Depth of Interaction Detector for PET," IEEE Trans Nucl Sci, Vol. 45, pp. 1078-1082, June 1998

[9] H. Murayama, H. Ishibashi, H. Uchida, T. Omura, and T. Yamashita, "Depth Encoding Multicrystal Detectors for PET," IEEE Trans Nucl Sci, Vol. 45, pp. 1152-1157, June 1998

[10] M. Dahlbom, L.R. MacDonald, L. Erikson, M. Paulus, M. Andreaco, M.E. Casey, C. Moyers, "Performance of a YSO/LSO Phoswich Detector for Use in a PET/SPECT System," IEEE Trans Nucl Sci, Vol. 44, pp. 1114-1119, June 1998

[11] M. Schmand, L. Erikson, M.E. Casey, D. Newport, K. Wienhard, W.D. Heiss, R. Nutt," Detector Design of a LSO Based Positron Emission Tomograph with Depth of Interaction Capability for High Resolution Brain Imaging," J Nucl Med, Vol. 39, p. 133P, May 1998

[12] GSO product sheet, Hitachi Chemical Company Ltd., Ibaraki, 312-0003 Japan

[13] J.J. Vaquero, J. Seidel, S. Siegel, W. R. Gandler, M. V. Green, "Performance Characteristics of a Compact Position-Sensitive LSO Detector Module," IEEE Trans Med Imag, Vol. 17 (6), pp. 967-978, 1998

[14] S. Nagai, M. Watanabe, H. Shimoi, H. Liu, and Y. Yoshizawa, "A New Compact Position-Sensitive PMT for Scintillation Detectors," Conference Record IEEE 1998 Nuclear Science Symposium \& Medical Imaging Conference 